\title{
Derrotas lacerantes y victorias deslumbrantes El imaginario nacional en Brasil a través de las Copas Mundiales
}

\author{
Painful defeats and dazzling victories \\ The national imaginary in Brazil through the World Cups \\ José Sérgio Leite Lopes* y Afrânio Garcia Jr. ** \\ Traducción del francés por María Virginia García \\ Revisión de la traducción por Ana Ines Heredia
}

RMA

Antropología Social

*Universidade Federal do Rio de Janeiro; **École des Hautes Études en Sciences Sociales

Traducción del texto "Défaites cuisantes et victoires éblouissantes L'imaginaire national au Brésil au prisme des Coupes du monde", publicado en Fabien Archambault, Stéphane Beaud y William Gasparini (Dir.), Le football des nations. Des terrains de jeu aux communautés imaginées. París, Publications de la Sorbonne, 2016, pp. 183-198.

\section{Negro, brasileño y futbolista: del polvo de arroz a la leyenda de Pelé}

En Francia, el Mundial 2014 provocó una serie de debates y reportajes que mostraba a un Brasil más complejo y problemático del que los franceses estaban acostumbrados a ver. También es cierto que la derrota de Brasil frente a Alemania por 7 a 1 en semifinales exhibió una Seleção completamente opuesta a la de sus días de gloria. Durante la primera semana de junio, antes de la ceremonia de apertura, el experimentado presentador del programa de televisión francés "C dans I'air" confesó haberse sorprendido al ver una película de Cantona sobre la historia, muy conocida en Brasil, de un jugador de "color" de Rio, de los años veinte, que se sintió obligado a cubrirse la cara con polvo de arroz para no abandonar el campo de juego. En efecto, son pocos los que recuerdan que antes de la victoria del Mundial de 1958, ser negro o mestizo era considerado una discapacidad para ingresar a la Seleção.

En Europa, Brasil está tan asociado al mestizaje y las virtudes del equipo brasileño a su carácter "arcoíris", que tornaban estos episodios incongruentes. Pero, nos estaríamos olvidando de que el fin de la esclavitud, en 1888, estuvo precedido por una política de "blanqueamiento de la raza", que promovió la inmigración masiva de europeos para borrar la huella de las "razas inferiores". El Ensayo sobre la desigualdad de las razas humanas de Arthur de Gobineau no tenía repercusión en Francia y sólo fue retomado por los alemanes durante el ascenso del nazismo, como lo recuerda el prologuista del volumen editado por la Pléiade, referencia que goza de prestigio bajo los trópicos.

\section{El fútbol y la valorización del mestizaje}

En Brasil, fueron muchos los "pensadores sociales" de fines del siglo XIX que citaban aquella referencia y varios ensayistas explicaban la inferioridad frente a la "civilización" con "teorías raciales". La población era mayoritariamente negra y mestiza porque los colonizadores portugueses, a partir del siglo XVI, aportaron contingentes predominantemente masculinos y al mismo tiempo, la cantidad de mujeres blancas era escasa, lo que significó una descendencia colonial mestiza. La jerarquía social se leía en los físicos de cada individuo, hombre o mujer. Hasta principios del siglo XX, la literatura de ensayo retomó formalmente la idea de los escritores racistas europeos de la "degeneración" de una población dominada por mestizos, incapaz, por naturaleza, de apropiarse de los beneficios de la "civilización" reconocida en el hemisferio norte.

Entre los años 1920 y 1960, las prácticas culturales como la literatura, el teatro, las artes plásticas, la música culta y popular y los deportes, como el fútbol, fueron sin duda las que contribuyeron a derribar el estigma de una población mayormente mestiza y a presentar este rasgo como una ventaja a la hora de enfrentar los desafíos de la modernización del país. Explicar las derrotas y las victorias en los Mundiales desde 1930 ocupa, evidentemente, un lugar preponderante al momento de verificar cómo la práctica del fútbol profesional permitió la valorización del mestizaje. Si bien esta "historia de éxitos" permitió en un principio calificarlo como el "país del fútbol" en 1958 y en 1962, hoy se topa con los efectos de las recientes derrotas sobre la autoestima de los hinchas y su repercusión bajo la forma de protestas en espacios públicos. La elaboración de la identidad nacional a través del fútbol está lejos de constituir un relato lineal y constructivo.

\section{De un deporte aristócrata a una práctica popular}

Los héroes emblemáticos de los Mundiales de 1958 y de 1962 -Garrincha y Pelé- no formaron parte del equipo inicial para la competición en Suecia. Recién ingresaron a jugar contra la URSS, en el tercero y decisivo partido para la clasificación, porque los mejores jugadores, Nilton Santos y Didi, presionaron al entrenador Vicente Feola. La marginación de estos jugadores se inscribía en una larga 
serie de prejuicios alimentada por la explicación de las derrotas en las competiciones anteriores: los jugadores negros y mestizos habrían sido los culpables. El éxito del jogo bonito y eficaz de Pelé y Garrincha, consagrados estrellas en ese momento, provocó el elogio de todo lo que antes se consideraba como prueba de incapacidad de algunas poblaciones para sobresalir en las competiciones promovidas por los "pueblos civilizados". Principalmente se aplaudieron las posturas corporales y la tendencia a privilegiar el carácter lúdico del juego.

La historia social del fútbol en Brasil y su inscripción en la escena internacional permite comprender cómo los prejuicios racistas tuvieron que someterse a los resultados de las competiciones internacionales quedándose sin fundamentos, al mismo tiempo que el deporte ampliaba su público y sus jugadores aficionados. La práctica de un deporte inicialmente importado por las élites aristócratas cedió progresivamente lugar a su apropiación por las clases populares, produciéndose el pasaje del amateurismo al profesionalismo. Si bien esto favoreció el flujo de jugadores de las clases populares, negros y mestizos en particular, las creencias y las prácticas racistas no desaparecieron del universo de ése deporte, adoptaron nuevas formas y se hicieron más fuertes luego de derrotas, como aquellas de los Mundiales de 1950 y 1954.

\section{La figura del "obrero jugador"}

Si el fútbol se hizo más popular en Inglaterra con la aparición de la Cup y del profesionalismo, la internacionalización de esta práctica deportiva sigue desde sus comienzos a una red de contactos que se produjo de manera indirecta por relaciones previas y espontáneas entre las élites y las instituciones inglesas de esa época. En Brasil, se observan partidos de fútbol que jugaban, en un principio, los marineros ingleses que estaban de paso o los empleados de empresas inglesas como por ejemplo las constructoras de las vías del tren.

Sin embargo, fueron los jóvenes de la élite brasileña que habían realizado sus estudios superiores en Inglaterra, los que construyeron la base de fundación de equipos de fútbol permanentes dentro de clubs preexistentes o la creación de nuevos clubs dedicados al fútbol. Por ejemplo, frecuentemente se considera a Charles Miller, ex estudiante de Southampton, hijo de un inglés y de una brasileña, como uno de los pioneros del fútbol por haber traído en sus valijas de regreso a São Paulo en 1894, dos pelotas de cuero y un uniforme completo. Y logró convencer a algunos socios del club de cricket del aristocrático São Paulo Athletic Club a que se inicien en este deporte.

Gracias a este tipo de iniciativas, varios de los clubs más selectivos terminaron reproduciendo en el campo de juego el microcosmos de las élites de Rio de Janeiro y de São Paulo. Además del cricket, algunos clubs practicaban remo. El Fluminense Football Club, creado en 1902 y primer club dedicado exclusivamente al fútbol, fue una referencia para las élites de Rio. Convocaba a un público elegante, los hombres iban de traje, corbata y sombrero, las jóvenes y las mujeres, de vestido elegante y sombrero de flores, como se acostumbraba en el Jockey Club. La pertenencia al mismo mundo social también se podía ver porque los jugadores solían frecuentar las tribunas durante los intervalos y al finalizar el partido para encontrase con sus familiares o su círculo de relaciones.

Paralelamente a los clubs selectivos, se desarrollaron clubs vinculados a fábricas textiles con barrios obreros en la periferia de las ciudades, como el club de Bangú, creado en 1904. Estos clubs reclutaban jugadores entre los obreros y, con el tiempo, la cantidad de jugadores negros y mestizos aumentó. En este marco se desarrolló la figura del "obrerojugador" que abriría la puerta a formas de profesionalismo disfrazado. Fue una de las compañías textiles, América Fabril, la que creó en 1908 el club que vio el ascenso del célebre Garrincha, "la alegría del pueblo", a principios de los años 1950 (Leite Lopes, Maresca, 1989).

\section{El polvo de arroz ridiculizado}

El crecimiento del profesionalismo disfrazado provocó violentos debates e incluso una escisión dentro de la Liga que organizaba los campeonatos en las metrópolis. Los clubs pioneros denunciaban una amenaza que recaía sobre el amateurismo como el "fin del juego por amor a la camiseta". En 1923, el club Vasco da Gama, donde también se practicaba remo pero que reagrupaba una numerosa colonia de inmigrantes portugueses, disputó por primera vez el campeonato de primera división y se consagró campeón. Su secreto: contratar a los mejores jugadores de los barrios de periferia, sean blancos, negros o mulatos y que entrenen a tiempo completo. A la inversa de los clubs aristocráticos, el Vasco no conformaba un equipo compuesto por individuos pertenecientes al mismo grupo social. La reacción de los otros clubs no se hizo esperar. Formaron otra liga y excluyeron al Vasco con la excusa de que no tenía estadio propio. Además, se implementó una prueba de alfabetización en la que los jugadores debían firmar antes de cada partido y completar una ficha de inscripción, marginando así a la gran mayoría proveniente de las clases populares. En semejante atmósfera se puede entender mejor que un jugador mulato, invitado a jugar en el aristocrático Fluminense, se haya sentido obligado a ponerse polvo de arroz sobre sus mejillas, lo que sus adversarios transformaron en burla entonando en cada partido "polvo de arroz".

\section{La emancipación de los negros por el deporte}

Sin embargo, el fútbol amateur brasileño estaba en crisis y las presiones internacionales le dieron el golpe de gracia. Durante los Mundiales, algunos jugadores que se sentían maltratados por los obstáculos que se les presentaban para ser profesionales, aceptaron ofertas de contrato provenientes del extranjero. Luego del Mundial de 1930, que ganó Uruguay, Mussolini con miras en la próxima competición en Italia cuatro años después, le dio su apoyo a los clubs italianos para reforzarse y éstos emprendieron 
una verdadera búsqueda de jugadores sudamericanos de origen italiano, principalmente en Argentina, Uruguay y Brasil. Frente al riesgo de perder a sus mejores jugadores el profesionalismo se reforzó. Hay que precisar que eran principalmente jugadores blancos los que iban a Europa, los negros eran excepciones, como fue el caso de Fausto, reconocido por sus habilidades en 1930. De todos modos, el profesionalismo terminó siendo un instrumento de emancipación de los negros, condición necesaria para que el fútbol adquiera estatus de deporte nacional.

Semejante proceso no implica solamente financiamiento sino también, la construcción de una proximidad, incluso de una identificación, entre los jugadores y el público unidos en un mismo proyecto de emancipación social por el deporte (Leite Lopes, 1994; Leite Lopes, Faguer, 1994). Esta identificación entre los jugadores y el público se produjo luego de que se implementara el profesionalismo. El Flamengo, que todavía era un club amateur, al contratar a jugadores negros de gran notoriedad como Fausto, Domingos da Guia y Leônidas da Silva, empezó a ser el club más popular de Rio. Los años 1930 fueron los de la democratización del fútbol, no solamente para los jugadores y los entrenadores, sino también desde el punto de vista de formación de un público que incluía a las masas populares. Durante la misma época, los trabajadores del campo empezaron a emigrar de las plantaciones agroindustriales a las grandes ciudades y la pasión por el fútbol contribuyó fuertemente a crear vínculos sociales de sustitución para estos individuos separados de sus familias y vecinos, limitados al medio urbano. Hablar de fútbol permitía fortalecer la comunicación entre personas de todos los orígenes.

\section{"Conquistar la hegemonía sobre ése deporte"}

La democratización del fútbol no terminó con los estereotipos de los negros y los mestizos. La derrota frente a Uruguay en el Maracanã en la final del Mundial 1950 provocó la estigmatización de los defensores negros de la Seleção, principalmente del arquero Moacir Barbosa y el stopper Bigode, considerados los responsables de la tragedia. Dirigentes deportivos y periodistas, mediante teorías raciales heredadas del evolucionismo del siglo XIX, le atribuyeron a los jugadores negros y mestizos una inestabilidad emocional más fuerte que los volvía ineptos para los partidos decisivos. Por ello, el mejor equipo de toda la competición, que había manifestado una gran habilidad en el manejo de la pelota frente a sus hinchas reunidos en el estadio más grande del mundo, prueba suplementaria del vigor de la industrialización del momento, había fracasado frente a un adversario técnicamente inferior, pero determinado a ganar y dueño de sus emociones. Las muchas músicas de carnaval que habían acompañado a cada una de las victorias, se transformó en un silencio agobiante.

En 1954, en Suiza, Hungría eliminó a Brasil con un resultado bastante amplio (4-2) y el partido terminó en una gran pelea. El jefe de la delegación João Lyra Filho publicó un informe en el cual atribuía la responsabilidad de la sucesión de derrotas a la composición racial del pueblo brasileño. Escribió: "A los jugadores brasileños les faltó lo que en general le falta al pueblo de Brasil. El estado psicosocial de nuestro pueblo todavía no ha madurado y los atletas que vienen del pueblo no pueden improvisar condiciones e instrumentos de superación durante estas pruebas deportivas que exigen la movilización de todos los recursos y todas las reservas orgánicas" (Guedes, 1998). La conclusión sobre el futuro de los brasileños en el fútbol era definitiva: "En el estado que se encuentra el pueblo brasileño, no podremos ser campeones del mundo y conquistar la hegemonía en este deporte". El catastrofismo movilizó a una comisión técnica, en la que figuraba un médico deportivo, que realizó un viaje a Europa para observar los métodos de preparación física para que los jugadores brasileños puedan competir con sus homólogos europeos en Suecia. En 1958 el virtuosismo del equipo nacional, sobre todo en la final y semifinal, dejaría boquiabierto a los apasionados del fútbol y haría olvidar las profecías pesimistas fundadas en prejuicios racistas.

\section{Pelé, 18 años y Garrincha, piernas torcidas, llamados como refuerzos}

Llama la atención cómo repercutieron las conclusiones del informe de Lyra Filho en la composición del equipo brasileño a principios de la competición. El único jugador negro era el mediocampista Didi, considerado por la prensa europea el mejor jugador del Mundial de 1958. Otros, como Garrincha y Pelé, fueron marginados por diferentes pretextos. Por ejemplo, un psicólogo diagnosticó a Garrincha como débil mental. Frente al riesgo de eliminación en el partido contra la URSS, los jugadores más experimentados del equipo, principalmente Nilton Santos y Didi, exigieron que fueran seleccionados el genial extremo derecho y el jovencísimo Pelé, que aún no cumplía los 18 años. La percepción de los atributos corporales de Garrincha, Pelé y Didi fue una revolución simbólica, invirtiéndose y transformándose en marcas de excelencia del fútbol.

Garrincha es quien mejor ilustra este cambio de estigmas corporales y sociales en capital físico y deportivo. Nació y creció en un barrio obrero textil, a 100 kilómetros de Rio, y compartía otros pasatiempos con los obreros, como la caza y la pesca. Con sus piernas torcidas y el desinterés que demostraba por todo lo que tenía que ver con su carrera, el mestizo Garrincha, auténtico representante de las clases populares brasileñas, constituye un caso ejemplar de la transformación de las discapacidades sociales en virtudes (Leite Lopes, Maresca, 1989).

La victoria de 1958, confirmada por el éxito en Chile en 1962, desmintió tajantemente los prejuicios que sostenían la incapacidad "natural" de los jugadores negros y mestizos para a afirmarse en las competiciones internacionales. En 1962, Pelé tuvo que abandonar la competición, que recién empezaba, a causa de una lesión; Garrincha se superó a sí mismo demostrando sus cualidades de goleador hasta entonces minimizadas. Además, los clubs de las dos estrellas, el Botafogo de Rio 
de Garrincha y el Santos de Pelé, tuvieron muchos éxitos en las competiciones nacionales e internacionales. Todas estas proezas contribuyeron a que el país se proyecte en la escena internacional y, como la música popular, reforzaron la confianza en la creatividad del pueblo, en un contexto de democratización de la sociedad brasileña, al menos hasta el golpe de Estado de 1964.

Sin embargo, la victoria en México en 1970 demostró la ambivalencia de las proezas de la Seleção como marcador de identidad asociado con modalidades de emancipación de fracciones de las clases populares por el deporte. De hecho, después de 1968, el régimen militar acentuó su rostro represivo generalizando la práctica de torturas como forma de control y de neutralización de sus oponentes. Por el contrario, para el fútbol los militares brindaron todos los medios materiales para conformar el mejor equipo posible. Con las expectativas puestas en ganar el Mundial de México, lanzaron una serie de slogans publicitarios y de canciones carnavalescas para motivar laexpresión de manifestaciones de alegría y reforzar así su legitimidad. Al principio, incluso aceptaron nombrar como técnico del equipo nacional a un periodista muy reconocido, João Saldanha, con un pasado comunista y convicciones de izquierda conocidos por todos - pero lo despidieron antes de salir para México. Una canción que se pasaba antes de la teledifusión de cada uno de los partidos entonaba: "De repente es un torrente que nos empuja hacia adelante / parece que todo Brasil se ha dado la mano / todos unidos con la misma emoción / todos, un solo corazón". La performance del equipo en México fue sensacional, derrotando a casi todos sus adversarios con cómodos resultados y Pelé jugó el mejor fútbol de su carrera. Además, gracias a los satélites, las cadenas de televisión pudieron difundir por primera vez los partidos en directo en todo el territorio. Por más que algunos grupos de izquierda mantuvieron cierta distancia con el equipo nacional, arguyendo el carácter represivo del Estado que la financiaba, la mayoría de los opositores comulgó con la Seleção. Las victorias se interpretaban como pruebas de las cualidades extraordinarias de las clases populares capaces de mostrarse superior al mundo, incluso en un contexto político desfavorable. Se impuso entonces la imagen de un fútbol considerado como una auténtica pasión popular, en donde las élites quedaban excluidas.

Además, los tres títulos de campeón del mundo contribuyeron a reforzar el sentimiento nacional. Se afirmó el estilo de juego que asociaba virtudes nacionales, principalmente del éxito de un mestizaje entre pueblos de diferentes continentes, que se oponía al hexis corporal de los europeos. Es decir, los rasgos característicos culturales de la colectividad nacional se inscribían en la técnica futbolística.

\section{La globalización del fútbol y la reapropiación por los intereses económicos}

Durante la época de oro, la mayoría de los jugadores se formaba en el país. Luego los compraban clubs europeos en donde terminaban sus carreras. Por lo tanto, la socialización de la práctica del deporte se realizaba en el país. La situación cambió cuando los medios financieros de los grandes clubs europeos aumentaron de manera considerable. A partir de la segunda mitad de los años ochenta y principios de los noventa, la mayoría de los jugadores que podía integrar la Seleção estaba en Europa. Progresivamente, se desarrolló un mercado secundario en Japón, Medio Oriente y Sudáfrica. No sólo partían jugadores que estaban terminando su carrera sino también entrenadores y miembros del cuerpo técnico (preparadores físicos, masajistas), contratados para mejorar a los equipos locales. Así, jugadores con experiencia y de gran nivel como Zagalo, Telê Santana o Zico entrenaron a las selecciones nacionales de países que debutaban en Mundiales.

A partir de 1990, el estudio de los efectivos de las selecciones nacionales de los Mundiales demostró que una parte considerable de ellas, casi la totalidad, estaban compuestas por jugadores que jugaban en clubs extranjeros y, en regla general, europeos. Brasil no escapaba a la regla. Una gran encuesta sobre los jugadores brasileños reclutados al extranjero, llevada a cabo por la antropóloga Carmen Rial, puso en evidencia algunos rasgos característicos de los candidatos que partían. En general, se trataba de menores, apoyados por la familia, convertidos al evangelismo y que querían volver al país una vez finalizada su corta carrera. El pase al extranjero les permitía acumular un patrimonio económico imposible de obtener si se quedaban en el país ${ }^{1}$.

La emigración, que cada vez se realizaba a una edad más joven, cambió profundamente la socialización de los jóvenes jugadores con la práctica deportiva. Hasta los años ochenta, la iniciación en el fútbol se hacía, en general, en escuelas de fútbol de clubs de divisiones inferiores de todo el país, muchas veces gracias a las modalidades mixtas entre amateurismo y profesionalismo. Este fenómeno de migraciones precoces rompió el vínculo entre las diferentes divisiones a escala nacional. La selección de una pequeña cantidad de jovencísimos jugadores contratados por los mejores clubs europeos creó un circuito restringido de jugadores con sueldos generoso y vació la fuente de renovación intergeneracional del país. El control creciente de los sponsors sobre la vida profesional y pública de los jugadores, alimentan al mercado mundial de estrellas abundantemente remuneradas. La derrota en la final del Mundial 1998 frente a Francia, da cuenta de estas tendencias.

De manera diferente a lo que ocurrió contra Uruguay en 1950 -el resultado parcial fue favorable para Uruguay recién en los últimos once minutos-, en 1998, la Seleção no jugó como lo había hecho en partidos anteriores,

\footnotetext{
La literatura sobre las migraciones de los africanos hacia Europa presenta constataciones similares.
} 
el primer tiempo terminó 2-0 en favor de Francia. El segundo tiempo fue todavía más deplorable y el resultado pudo haber sido más amplio, como lo remarcó un Pelé horrorizado en la cadena de televisión Globo que lo había contratado como comentarista. Todos los jugadores tuvieron una mala presentación pero esta vez no se buscó a ningún chivo expiatorio como en 1950. Sin embargo, visto desde Brasil, los problemas de salud que tuvo Ronaldo antes de que empiece el partido contribuyeron, sin duda, a debilitar la moral de todo el equipo.

Ronaldo, conocido en esa época como "el fenómeno", tenía una trayectoria parecida a la de Pelé. Originario de clases populares de la periferia de Rio, jugaba fútbol de salón en un club de baja categoría, São Cristóvão, y tenía muchas dificultades para financiar su "pasión". Conoció a uno de los grandes campeones de 1970, Jairzinho, que le propuso ser su manager. Éste, convenció a los funcionarios de la Confederación Brasileña de Fútbol (CBF) que llamaron a Ronaldo para que forme parte de la selección nacional sub 17. Al igual que Pelé, Ronaldo fue seleccionado a una edad precoz para formar parte del equipo que participaría en la Copa del Mundo de 1994. Si bien no jugó, antes de que termine la Copa fue transferido de un buen club de Minas Gerais, en el centro de Brasil -el Cruzeiro-, al PVS Eindhoven. Luego, se fue a jugar al FC Barcelona y en 1998, al Inter de Milán, Firmó varios contratos de publicidad con diferentes sponsors, fascinados con la idea de apostar por un joven que prometía una larga carrera llena de éxitos. Primero firmó con Brahma, una marca de cerveza muy conocida en Brasil. Luego, con firmas internacionales como Nike, Pirelli y Parmalat. Nike dejaba en ese momento sus inversiones en los equipos de básquet para concentrarse en el fútbol y le propuso a Ronaldo un contrato vitalicio. Para los hinchas brasileños el itinerario de Ronaldo simbolizaba las contradicciones del fútbol globalizado, altos salarios y en contrapartida, el encadenamiento a entrenamientos, partidos y presentaciones en la televisión o en la radio a un ritmo desenfrenado.

En 1998, la noche antes de la final, Ronaldo tuvo una fuerte convulsión. Roberto Carlos, su compañero de cuarto, fue quien advirtió a los dirigentes y el atacante fue trasladado a la guardia de un hospital parisino. Las cadenas brasileñas que transmitirían el partido en directo, anunciaron que Ronaldo no jugaría la final, pero no dijeron por qué.. Al día siguiente de la final, la prensa de Rio difundió el contenido de una entrevista a un importante neurólogo brasileño, Paulo Niemeyer, que fue muy virulento con los dirigentes de la CBF y pidió la suspensión del médico de la Seleção. Según él, Ronaldo tendría que haber hecho reposo total, ya que estaba totalmente incapacitado para jugar la final de un Mundial. Y agregó que el jugador podría haber sufrido un accidente cerebral más grave, incluso mortal, lo que transformaba a la CBF en una banda de asesinos en potencia. Todas las miradas cayeron sobre Nike y las cláusulas secretas que tenía con la Federación brasileña.
Por ello, en el proceso de identificación de los responsables de la derrota, a diferencia de lo que ocurrió en 1950, esta vez no fueron los jugadores los señalados con el dedo sino los dirigentes. Ronaldo, personaje central del drama de una derrota inexplicable, se percibía como la pieza rota de un engranaje más amplio controlado por una administración incompetente que realizaba negocios turbios y que no publicaba ni sus ingresos ni sus gastos. Se organizaron dos comisiones revisoras parlamentarias -una en la Cámara de diputados y otra en el Senado- para revelar la verdad sobre los contratos firmados entre la CBF y Nike. Hay que tener en cuenta que las manifestaciones de protesta contra las modalidades de organización del Mundial 2014 se inscriben en una herencia de sospechas hacia los dirigentes deportivos y por lo tanto, hacia los políticos.

\section{Crónica de una catástrofe anunciada: el Mundial 2014}

En el 2014 cuando Brasil fue sede, por segunda vez, de la Copa Mundial de Fútbol todos esperaban que el comité organizador no esté a la altura de las circunstancias. Sin embargo, las previsiones catastróficas de los oponentes al Partido de los Trabajadores fueron desmentidas. Desde el principio de la competición, fueron reemplazadas por los elogios unánimes de los turistas y periodistas del mundo entero. La Seleção era favorita dado que el año anterior había vencido en la final de la Copa Confederaciones a España, último campeón del mundo. La derrota frente a Alemania con el humillante resultado 7-1, con cinco goles en el primer tiempo y todo el equipo completamente sobrepasado por los hechos, incapaz de reaccionar, jamás se había visto. La derrota demostró la fragilidad de la construcción de la autoestima nacional fundada únicamente en la competitividad deportiva. Y aún fue más sorprendente la negación radical que se le dio al viejo refrán que califica al fútbol como "el opio del pueblo". En junio de 2013, cuando se entregaron las primeras instalaciones deportivas, protestas masivas llenaron las calles en todos los estados de Brasil. Se reunían reivindicaciones de carácter político sobre carencias en materia educativa, salud, transporte público y se criticaban las infraestructuras destinadas a los atletas e hinchas extranjeros. Sin nombrar las denuncias de corrupción tanto a los dirigentes brasileños como a los de la FIFA y las empresas de trabajos públicos beneficiarias de contratos gigantescos. La organización del Mundial 2014 fue el catalizador de reivindicaciones populares totalmente alejadas de las preocupaciones deportivas. Hubo que desprenderse de los estereotipos que relacionan al fútbol y la política en Brasil.

Estas manifestaciones fueron el comienzo de una crisis política que hoy todavía dura y que las elecciones presidenciales, legislativas, de senadores y regionales no pudieron aplacar. Durante el primer semestre del 2013, estudiantes de secundario y de la universidad manifestaron en São Paulo reclamando la gratuidad del 
transporte público. Los enfrentamientos con la policía se hicieron cada vez más frecuentes y violentos. La brutalidad de la represión de la policía, en lugar de debilitar a la movilización, acarreó la protesta de otras categorías sociales que denunciaban los modos de organización y contratación de efectivos policiales señalando que no había cambiado nada desde fines del régimen militar. Las escenas filmadas gracias a los teléfonos celulares y la difusión en internet alimentaron aún más las protestas. El abanico de consignas indicaba que entre las millones de personas que salieron a las calles había desde estudiantes de secundario y universitarios, hasta militantes de extrema izquierda y de extrema derecha, algunos de estos últimos pedían abiertamente el regreso de los militares al poder. También salieron a las calles diferentes fracciones de la clase media, disgustadas por las políticas sociales destinadas a disminuir la pobreza y preocupadas por mantener su jerarquía social y su estatus privilegiado. Pero también manifestaron sindicalistas, miembros de asociaciones de barrios desfavorecidos, como por ejemplo los militantes del movimiento "sin tierra" y "sin techo". Los partidos políticos y los delegados no fueron bien recibidos. Parecía que otra estructura del espacio público debiera renovar totalmente a las instituciones políticas. Dilma Rousseff propuso un debate en torno de la agenda de reformas políticas, pero la clase política, incluso dentro de su familia política, simplemente esperó que el mar de manifestantes se extinga y las múltiples reivindicaciones queden en el olvido.

Es imposible analizar aquí los múltiples significados de este cuestionamiento de las instituciones políticas de la Constitución de 1988. Por el contrario, hay que resaltar, siguiendo a los antropólogos Arlei Damo y Ruben Oliven (Damo, Oliven, 2013), que la organización del Mundial generó un debate político por fuera de las estructuras tradicionales de la vida política.

\section{Conclusión}

Para concluir, recordaremos el golpe que generó la puesta en marcha del pliego de condiciones de la FIFA. La construcción de infraestructuras reveló la distancia que hay entre las normas y los estándares internacionales y la realidad brasileña. Primero, la prensa juzgó que estas condiciones llevarían a Brasil a modernizarse. Sin embargo, progresivamente se reveló el reverso de la moneda. La FIFA tenía ante todo, exigencias contrarias a las prácticas locales, como la reintroducción forzada de cerveza en botellas en los estadios o la prohibición de pequeños comercios a los alrededores del recinto. Además, la Federación internacional era el único juez de cómo y qué se debía construir. En todo el país, crecía un sentimiento de despojo de una parte de la soberanía nacional. Los estadios que se construyeron no tenían nada que ver con los que se habían hecho en 1950 y esto trajo consecuencias en la morfología social del público deportivo. Durante el anterior Mundial organizado en Brasil, el Maracanã de Rio, el "estadio más grande del mundo" había destinado un lugar privilegiado para los espectadores humildes que se sentaban directamente sobre el cemento ("arquibancadas") y sobre todo para el público que miraba el partido de pie ("gerais"). Por el contrario, en 2014 la FIFA construía los estadios de fútbol como si fueran un teatro o un cine, de los que se excluía a las masas populares. De allí venía el sentimiento de ser despojado de su propia cultura en beneficio de las clases sociales más acomodadas. El debate causó estragos. ¿Quién se quedaría después del Mundial con las instalaciones "standard FIFA"? ¿De qué manera, los clubs que no llegaban a pagar regularmente a sus jugadores o a impedir que las nuevas generaciones de jóvenes prometedores estén condenados a emigrar a una edad precoz, podrían financiar el mantenimiento de estas infraestructuras? Gran paradoja que las fuerzas populares, una vez en el poder, vire la "pasión nacional" en beneficio de las clases más acomodadas, siguiendo las imposiciones de la FIFA y en connivencia con los sponsors multinacionales y los intereses de una prensa completamente dependiente de los grandes grupos económicos y financieros.

París / Río de Janeiro 2016

\section{Referencias bibliográficas}

Da Matta,. R. (1979). Carnavais, malandros e heróis. Rio de Janeiro, Zahar.

Damo, A., Oliven, R. (2013). "O Brasil no horizonte dos megaeventos esportivos de 2014 e 2016". Horizontes antropológicos, $\mathrm{n}^{\circ} 40$, pp. 19-63.

Filho, M. (2003 [1 ${ }^{\text {ra }}$ ed. 1947]). O negro no futebol brasileiro, Rio de Janeiro, Mauad.

Garcia Jr., A. (1993). Les intellectuels et la conscience nationale au Brésil, Actes de la recherche en sciences sociales, 98:20-33.

Guedes, S. (1998), O Brasil no campo do futebol. Estudos antropológicos sobre os significados do futebol brasileiro. Niterói, EDUFF.

Leite Lopes, J. S., Maresca, S. (1989). La disparition de la "joie du peuple", Actes de la recherche en sciences sociales, 79: 2136.

Leite Lopes, J. S., Faguer, J.P. (1994). " L'invention du style brésilien. Sport, journalisme et politique ». Actes de la recherche en sciences sociales, $n^{\circ} 103, \mathrm{pp}$. 27-35.

Leite Lopes, J. S. (2004). "Classe, etnicidade e cor na formação do futebol brasileiro". En Cláudio Batalha, Fernando Teixeira Da Silva, Alexandre Fortes (dir.), Culturas de classe: identidade e diversidade na formação do operariado, Campinas, Unicamp, pp. 121-163.

Leite Lopes, J. S. (2014). "Transformações na identidade nacional construída através do futebol: lições de duas derrotas históricas". En João Whitaker Ferreira (dir.), Brasil em jogo, o que fica da Copa e das Olimpíadas? São Paulo, Boitempo, pp. 41-49. 